\title{
The Scratch Collapse Test Supported the Diagnosis and Showed Successful Treatment of Tarsal Tunnel Syndrome
}

\author{
Ibrahim Turan $^{\mathrm{a}}$, Elisabet Hagert ${ }^{\mathrm{b}}$, Jan Jakobsson ${ }^{\mathrm{c}, \mathrm{d}}$
}

\begin{abstract}
We describe a case where the scratch collapse test was found positive for tarsal tunnel syndrome and negative after successful surgery in a female patient.
\end{abstract}

Keywords: Tarsal tunnel; Scratch collapse test; Diagnosis; Nerve entrapment

\section{Introduction}

Peroneal or fibular neuropathy is a frequent mononeuropathy encountered in the lower limb [1]. The tarsal tunnel syndrome (TTS) is a rare but important condition which is regularly under diagnosed leading to a range of symptoms affecting the plantar aspect of the foot. It is an entrapment neuropathy of the posterior tibial nerve or its branches within its fibro-osseous tunnel beneath the flexor retinaculum on the medial side of the ankle. The symptoms consist of pain/ paresthesia, neural hyperalgesia, sensory loss, and dysesthesia and may also include weakness. Physical examination includes a positive Tinel's test and if not handled appropriate atrophy. Tinel's sign is a classic way to detect irritated nerves, performed by lightly tapping (percussing) over the nerve to elicit a sensation of tingling/paresthesia along the distribution of the nerve involved [2]. The exact cause is not known. Physical exercise, highly repetitive and rapid foot

\footnotetext{
Manuscript accepted for publication August 12, 2013

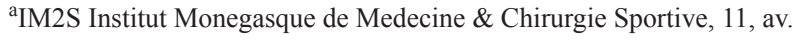
d'Ostende MC 98000, Monaco

${ }^{\mathrm{b}}$ Hand \& Foot Surgery Center, Storangsv.10, Stockholm, Sweden

${ }^{\mathrm{c}}$ Department of Anaesthesia, Karolinska Institutet, Danderyds

University Hospital, Stockholm, Sweden

${ }^{\mathrm{d}}$ Corresponding author: Jan Jakobsson, Department of Anaesthesia,

Danderyds Hospital, 18288 Danderyd, Sweden.

Email: jan.jakobsson@ki.se
}

doi: http://dx.doi.org/10.4021/jmc1453w motions, is a known risk factor. Diabetes, arthritis, hormonal disorders, and pregnancy may also increase risk. Varicose vessels, ganglion, and edema are commonly found at exploration.

\section{Case Report}

We here describe a case, a 54-year-old woman with no previous medical history complaining about exercise-induced pain from the medial malleolus and distal since a couple of weeks. The symptoms had increased during the last week, especially following walking and/or running. She denied having had any trauma. Examination showed typical palpation pain medial and over the lateral corner of talus within the talocrural joint in the ankle. There were also crepitations during flexion, but no signs of instability. No pain when palpating the peroneus tendon. The scratch collapse test, which entails firm scratching over the tarsal tunnel followed by resisted external rotation of the arm, revealed loss of ipsilateral arm strength. MRI showed no pathology and the EMG was unspecific. The patient was scheduled for elective surgery release of the nerve. The patient had elective surgery and the nerve was decompressed according to the technique described by Turan et al [3]. Surgery revealed a classical compression and the entire area was decompressed according to the technique described. Surgery was uncomplicated and already following early recovery when re-examining the scratch collapse test 30 min after surgery, it was found negative. When strength was tested, there was clear and obvious strength, a finding contrary to the preoperative SCT, which resulted in loss of strength in isometric external rotation of the ipsilateral arm.

\section{Discussion}

Cheng et al described in 2008 the high sensitivity and specificity for the use of SCT in diagnosis of carpal tunnel syndrome and cubital tunnel syndrome [4]. The scratch collapse test is done by placing the patient in a seated position with knees flexed to $90^{\circ}$. The patient's elbows are placed at the 
side and flexed to $90^{\circ}$. The patient was instructed to resist the force applied by the examiner. With the patient resisting, the examiner's hands were placed on the patient's forearms and an equal force was applied bilaterally in an inward direction. Thus, the patient applied a force of external rotation to equal the examiner's force of internal rotation. The patient was then asked to hold the elbow-flexed position as the examiner gently stroked, or "scratched," the skin surface once over the path of the peroneal nerve at the fibular neck. The examiner once again instructed the patient to perform resisted external rotation. A positive test was noted by ipsi-lateral weakness of resisted external rotation and collapse of the arm inward. Gillenwater et al described the scratch collapse test being a sensitive and specific provocative test that compares favorably to existing clinical tests and aids in the diagnosis of common peroneal neuropathy [5]. The mechanism behind the scratch collapse test is not known still taking into account that it is easy, simple, and completely harmless to perform the benefit versus risk for its use seems positive.

Tarsal tunnel syndrome is not uncommonly miss-diagnosed. It is not uncommonly mistaken for Achilles tendonitis, plantar fasciitis or calcaneal spur. The results of surgery are dependent on early treatment and thus diagnostic delay may cause unfavorable long-term effects. This is as far as we know the first experience with the scratch collapse test used as an examination to verify the nerve dysfunction and rapid recovery after early corrective surgery. We have since this first patient experienced similar positive scratch collapse test in two additional patients. The patho-physiology is similar to the carpal tunnel syndrome and similar nerve entrapment on the arm [6]. Brown et al found that the scratch collapse test was a seemingly reliable physical examination technique for localizing the point of maximal nerve compression in patients with cubital tunnel syndrome [7]. The scratch collapse test is simple and rapid to perform and it seems reasonable to add this test to the diagnostic toolbox also for analysis of ankle pain. It may also be a simple way to evaluate surgical effect already shortly after surgery, before sending patient home.

\section{Conflict of Interest}

The authors have no conflicts of interest.

\section{References}

1. Marciniak C. Fibular (peroneal) neuropathy: electrodiagnostic features and clinical correlates. Phys Med Rehabil Clin N Am. 2013;24(1):121-137.

2. Henderson WR. Clinical assessment of peripheral nerve injuries; Tinel's test. Lancet. 1948;2(6534):801-805.

3. Turan I, Rivero-Melian C, Guntner P, Rolf C. Tarsal tunnel syndrome. Outcome of surgery in longstanding cases. Clin Orthop Relat Res. 1997;(343):151-156.

4. Cheng CJ, Mackinnon-Patterson B, Beck JL, Mackinnon SE. Scratch collapse test for evaluation of carpal and cubital tunnel syndrome. J Hand Surg Am. 2008;33(9):1518-1524.

5. Gillenwater J, Cheng J, Mackinnon SE. Evaluation of the scratch collapse test in peroneal nerve compression. Plast Reconstr Surg. 2011;128(4):933-939.

6. Hagert E. Clinical diagnosis and wide-awake surgical treatment of proximal median nerve entrapment at the elbow: a prospective study. HAND. 2013;8(1):41-46.

7. Brown JM, Mokhtee D, Evangelista MS, Mackinnon SE. Scratch Collapse Test Localizes Osborne's Band as the Point of Maximal Nerve Compression in Cubital Tunnel Syndrome. Hand (N Y). 2010;5(2):141-147. 